\title{
MODELOS DE ESTIMATIVA DA RADIAÇÃO FOTOSSINTETICAMENTE ATIVA GLOBAL E DIFUSA EM FUNÇÃO DA RADIAÇÃO DE ONDAS CURTAS E DO ÍNDICE DE CLARIDADE (Kt)
}

\author{
Eduardo Nardini Gomes \\ João Francisco Escobedo \\ Departamento de Recursos Naturais, Faculdade de Ciências Agronômicas, Universidade Estadual \\ Paulista, CP 237, CEP 18603-970, Botucatu - SP, Fone: (0xx14) 6802-7162
}

\section{RESUMO}

O presente trabalho apresenta equações de estimativa da radiação fotossinteticamente ativa global $\left(H_{G p}^{d}\right)$ e difusa $\left(H_{d p}^{d}\right)$ em função das respectivas radiações global $\left(H_{G}^{d}\right)$ e difusa $\left(H_{d p}^{d}\right)$ do espectro solar total, bem como a estimativa da fração PAR difusa da PAR global $\left(K_{d p}^{d}\right)$ em função do índice de transmissividade atmosférica $\left(K_{t}^{d}\right)$.

A base de dados foi adquirida no período de 01/06/1999 a 31/09/2000 na Estação de Radiometria Solar da FCA-UNESP, Botucatu. Foram utilizados dados adicionais, diferentes dos utilizados na geração dos modelos, de forma a possibilitar uma validação adequada dos modelos propostos.

UNITERMOS: radiação fotossinteticamente ativa global e difusa, transmissividade atmosférica, modelos de estimativa da radiação solar.

\section{GOMES, E.N., ESCOBEDO, J.F MODELS FOR GLOBAL AND DIFFUSE PHOTOSYNTHETICALLY ACTIVE RADIATION IN RELATION TO GLOBAL, DIFFUSE RADIATION AND CLEARNESS INDEX.}

\section{SUMMARY}

This work describes typical correlations between global solar radiation $\left(H_{G}^{d}\right)$ and its global PAR component $\left(H_{G p}^{d}\right)$, diffuse solar radiation $\left(H_{d}^{d}\right)$ and its diffuse PAR component $\left(H_{d p}^{d}\right)$, clearness index $\left(K_{t}^{d}\right)$ and the diffuse PAR fraction of global PAR $\left(K_{d p}^{d}\right)$. Database was recorded from June $1^{\text {st }} 1999$ to September $31^{\text {st }} 2000$ at the Solar Radiometric Station, Botucatu, SP. Additional data which are not part of the model development were used to validate each proposed model.

KEYWORDS: global and diffuse photosynthetically active radiation, clearness index, estimating models. 


\section{INTRODUÇÃO}

Os modelos de crescimento de plantas requerem a precisa estimativa da fotossíntese, e para isto, a energia radiante interceptada é um importante parâmetro a ser considerado em qualquer algoritmo de fotossíntese. A radiação fotossinteticamente ativa (PAR) é o termo genérico que define a energia radiante nos limites de 0,4 a 0,7 $\mu \mathrm{m}$ do espectro solar (Monteith, 1972). Segundo Alados \& AladosArboledas (1999), o conhecimento PAR (Photosynthetically Active Radiation) se faz necessário em diferentes aplicações, sendo requerida na estimativa da luz interceptada para fins de modelagem de fotossíntese em comunidades de plantas simples e complexas. Alvalá \& Silva (2000) afirmaram que a PAR se faz necessária também na caracterização do crescimento e morfologia de plantas, bem como na estimativa de competição e avaliação da interação entre elas, apresentando também destacada importância na modelagem de florestas (Hassika \& Berbigier 1998), em ambiente protegido (Frisina et al. 2000) e em modelagem ecológica (Brock, 1981; Silva \& Asmus, 2001).

Em geral, a PAR não é medida de rotina nas estações meteorológicas, e como alternativa de conhecimento dos níveis de energia desta radiação, vários trabalhos apresentam equações de estimativa da PAR global (representada pela soma da PAR direta na horizontal com a PAR difusa) através de diversos parâmetros que são medidos de rotina na maioria das estações meteorológicas e radiométricas mundiais (Mccree, 1966; Yocum et al., 1969; Britton \& Dodd, 1976; Stigter \& Musabilha, 1982; Howell et al., 1983; Papaioannou et al. 1993), e alguns destes apontam para a necessidade de uma calibração local da relação entre a PAR e a radiação global, devido a diferenças climáticas locais. Contudo, pouca atenção tem sido direcionada às componentes direta e difusa deste fluxo radiométrico, devido principalmente a limitações de natureza instrumental, bem como devido a sua dependência com as condições do tempo e da atmosfera (Karalis, 1989), necessitando também de técnicos mais especializados que as medições convencionais da PAR global.

Devido a maioria das estações meteorológicas existentes não disporem de muitos sensores e ao mesmo tempo, necessitarem estimar componentes que não podem ser medidas de rotina, desenvolveu-se o índice $K_{d p}^{d}$, que representa a fração PAR difusa da PAR global, sendo este índice empregado no desenvolvimento de um modelo polinomial para a estimativa de $K_{d p}^{d}$ com base nos valores da transmissividade atmosférica diária $\left(K_{t}^{d}\right)$, visando possibilitar a estimativa da componente PAR difusa $\left(H_{d p}^{d}\right)$ através dos valores medidos da componente PAR global $\left(H_{G P}^{d}\right)$.

Os objetivos deste trabalho são: relacionar as componentes global e difusa da PAR com as radiação de ondas curtas na partição diária. Ajustar modelos de estimativa entre PAR global e radiação global, PAR difusa e radiação difusa, e fração PAR difusa da PAR global $\mathrm{e} o$ índice de transmissividade atmosférica e validar os modelos propostos.

\section{MATERIAL E MÉTODOS}

A base de dados diária utilizada neste trabalho é composta por medidas diretas e indiretas de determinadas componentes da radiação solar, monitoradas de junho de 1999 a maio de 2000 na Estação de Radiometria Solar, UNESP-Campus de Botucatu, localizada na latitude de $22^{\circ} 51^{\prime}$ Sul, longitude $48^{\circ} 27^{\prime}$ 'Oeste e altitude $786 \mathrm{~m}$. O clima local é típico das regiões subtropicais do Brasil, com inverno seco e verão úmido, e segundo a classificação de Köppen é do tipo Cwa. A temperatura e precipitação pluvial médias anuais são respectivamente iguais a $20,5{ }^{\circ} \mathrm{C}$ e $1533,2 \mathrm{~mm}$.

$\mathrm{Na}$ geração e validação dos modelos propostos, utilizou-se de uma base de dados de 16 meses do período de junho de 1999 a setembro de 2000. Em cada conjunto de quatro meses consecutivos, os três primeiros meses foram selecionados e utilizados na geração dos 
modelos e o mês restante na validação. $\mathrm{Na}$ busca de possíveis erros na coleta, armazenamento ou na transferência dos dados, os valores de densidade de fluxo de todas as componentes foram individualmente verificados. Os valores negativos e os zeros absolutos foram excluídos

As irradiâncias global $\left(I_{G}\right)$ e PAR global

$\left(I_{G P}\right)$ foram monitoradas através de um piranômetro EPPLEY-PSP $\left(\mathrm{K}=8,13 \mu \mathrm{V} / \mathrm{Wm}^{2}\right)$ e um sensor quântico da LI-COR $\left(\mathrm{K}=10,63 \mu \mathrm{V} / \mathrm{Wm}^{2}\right)$ e as irradiâncias direta $\left(I_{G}\right)$ e PAR direta na incidência $\left(I_{b p}\right)$ por pireliômetro EPPLEY-NIP $\left(\mathrm{K}=7,73 \mu \mathrm{V} / \mathrm{Wm}^{2}\right) \mathrm{e}$ um pireliômetro com sensor quântico da LICOR $\left(\mathrm{K}=14,14 \mu \mathrm{V} / \mathrm{Wm}^{2}\right)$, ambos acoplados a um rastreador solar EPPLEY modelo ST-3. As irradiâncias direta na horizontal $\left(I_{D H}\right)$ e PAR direta na horizontal foram obtidas através da multiplicação das irradiâncias $I_{b}$ e $I_{b p}$ pelo coseno do ângulo zenital instantâneo, e integradas diariamente de forma a possibilitar a obtenção das medidas indiretas da difusa e PAR difusa pelo método da diferença.

No trabalho, as irradiações estão representadas por $H_{X}^{d}$, onde $\mathrm{d}$ representa $\mathrm{o}$ intervalo de tempo (de integração das irradiâncias) de 1 dia e $\mathrm{X}$ representa cada radiação (G para global, b para direta na incidência, DH para direta na horizontal, $\mathbf{d}$ para difusa, Gp para PAR global, bp para PAR direta na incidência, DHp para PAR direta na horizontal, dp para PAR difusa e $\mathbf{O}$ para a radiação no topo da atmosfera).

A irradiação difusa diária $\left(H_{d}^{d}\right)$ foi obtida pelo método da diferença entre as radiações global $\left(H_{G}^{d}\right)$ e direta na horizontal $\left(H_{D H}^{d}\right)$, ou seja: $H_{d}^{d}=H_{G}^{d}-H_{D H}^{d}$, o mesmo ocorrendo com a PAR difusa $\left(H_{d p}^{d}\right)$ pela da diferença entre PAR global $\left(H_{G p}^{d}\right)$ e PAR direta na horizontal $\left(H_{D H p}^{d}\right)$, através da expressão: $H_{d p}^{d}=H_{G p}^{d}-H_{D H p}^{d}$. Obteve-se o índice de transmitividade atmosférica $\left(K_{t}^{d}\right)$ através da expressão: $K t=H_{G}^{d} / H_{O}^{d}$.

A avaliação da performance dos modelos se deu através dos indicativos estatísticos MBE (Mean Bias Error), RMSE (Root Mean Square
Error), índice de correlação (r), $d$ de Willmott (1981). Segundo Willmott (1981), somente o uso do coeficiente de correlação (r) ou do coeficiente de determinação $\left(\mathrm{r}^{2}\right)$, conjuntamente com testes sobre sua significância estatística, são inadequados na avaliação de modelos. Se por um lado os coeficientes $r$ e $r^{2}$ descrevem bem consistentes acréscimos e decréscimos proporcionais sobre as respectivas médias de duas variáveis, por outro lado eles não fazem distinção entre tipo e magnitude de possíveis covariâncias.

O indicativo estatístico MBE representa o desvio das médias, e provê informações quanto a performance dos modelos a longo prazo, informa também quanto a subestimativa ou superestimativa, mas não informa sobre espalhamento. Uma desvantagem deste indicativo é que uma subestimativa individual anula uma superestimativa.

O grau de espalhamento obtido na correlação entre os valores estimados e medidos é observável no indicativo estatístico RMSE, já o indicativo estatístico $d$ indica o quanto a reta ajustada através dos valores estimados e medidos se aproxima da reta ideal (1:1).

$O$ uso conjunto dos indicativos estatísticos RMSE e $d$ tem-se demonstrado um índice superior e de adequada significância estatística, como afirmado por Willmott (1981). Alados et al.(2000) utiliza o índice $d$ conjuntamente com o RMSE no teste de validação de seus modelos de radiação solar.

\section{RESULTADOS E DISCUSSÃO}

As Figuras 1 e 2 ilustram a correlação entre as componentes $H_{G}^{d}$ e $H_{G p}^{d} ; H_{d}^{d}$ e $H_{d p}^{d}$ na partição diária, bem como as retas ajustadas através de regressão linear simples entre 336 pontos de medidas simultâneas entre todas estas radiações, originando os modelos expressos por:

Modelo I: $\quad H_{G p}^{d}=0,5183^{*} H_{G}^{d}$

Modelo II: $\quad H_{d p}^{d}=0,6469 * H_{d}^{d}$ 
A Figura 3 mostra a correlação entre o índice de transmissividade atmosférica diária $\left(K_{t}^{d}\right)$ e a fração PAR difusa da PAR global $\left(K_{d p}^{d}\right)$ bem como a curva polinomial ajustada através de 336 pontos de medidas simultâneas entre todas as radiações que compõe os índices, originando o modelo expresso por:

Modelo III: $K_{d p}^{d}=\left[1,05142-\left(0,14987^{*} K_{t}^{d}\right)\right.$ -

$$
\left.1,46649 *\left(K_{t}^{d}\right)^{2}\right] \text {. }
$$

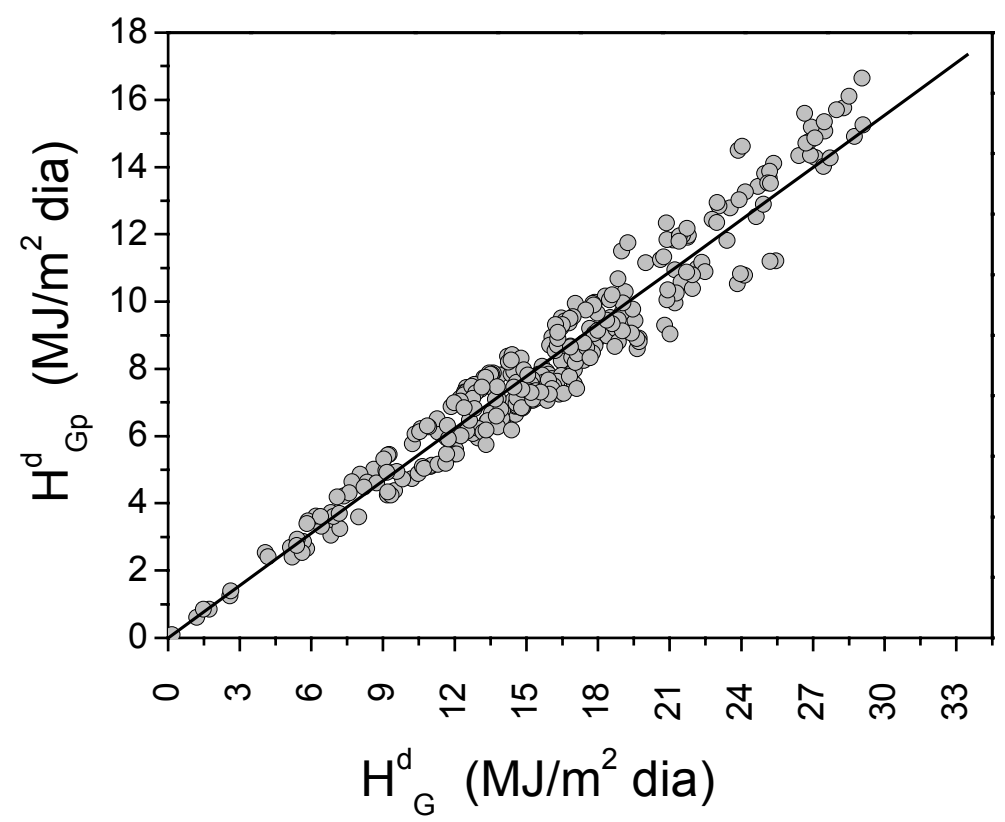

Figura 1-Correlação entre $H_{G}^{d}$ e $H_{G p}^{d}$ e reta ajustada através de regressão linear simples.

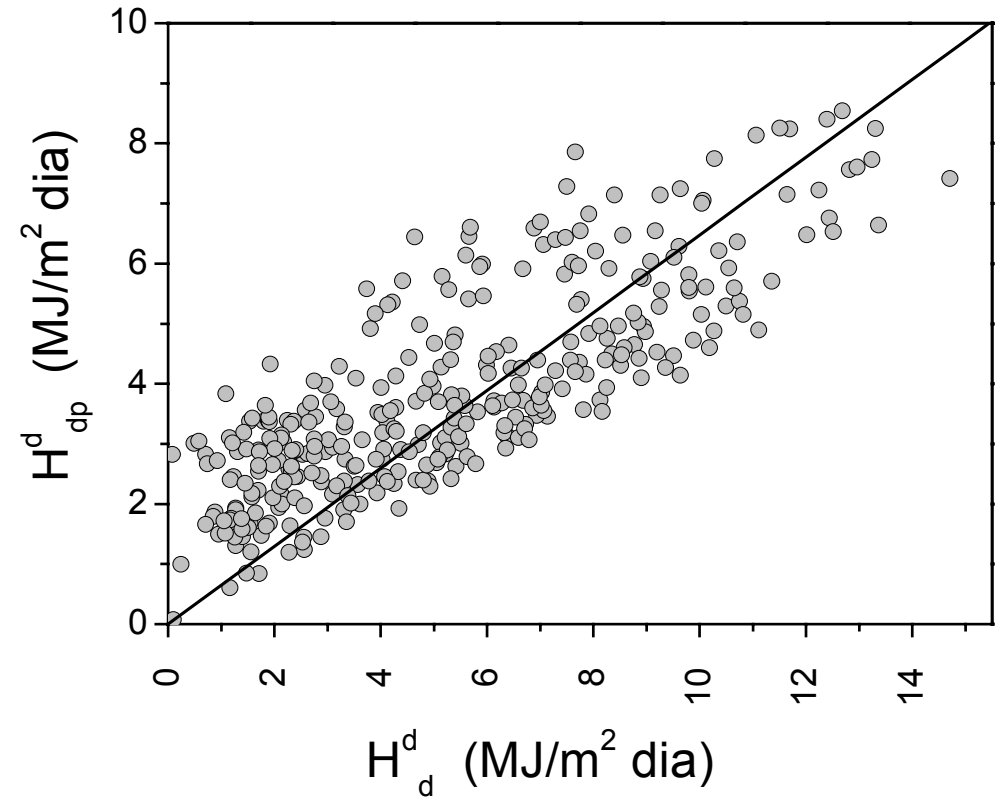

Figura 2-Correlação entre $H_{d}^{d}$ e $H_{d p}^{d}$ e reta ajustada através de regressão linear simples. 


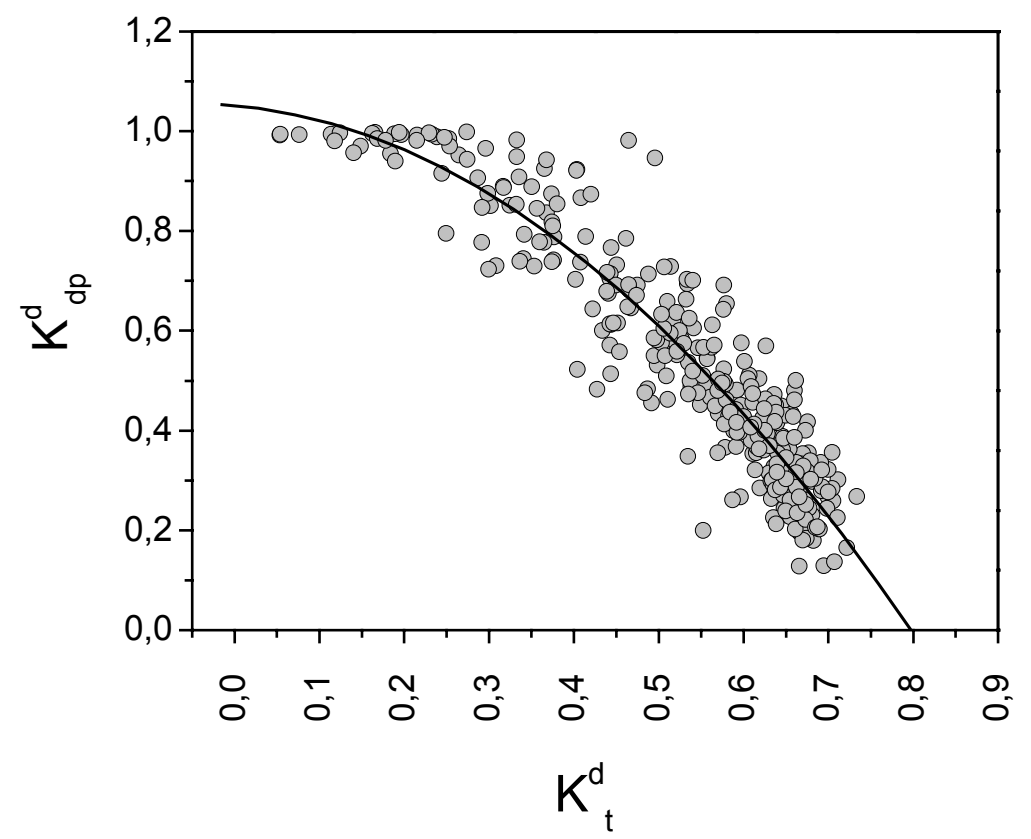

Figura 3-Correlação entre $K_{d p}^{d}$ e $K_{d p}^{d}$ e curva polinomial ajustada.

A validação dos modelos diários através dos indicativos estatísticos MBE, RMSE, $r$ e $d$ de Willmott dos modelo I, II e III está expressa no Quadro 1:

Quadro 1. Coeficiente angular e indicativos estatísticos de correlação.

\begin{tabular}{ccccc}
\hline & MBE (\%) & RMSE (\%) & r & d (Willmott) \\
\hline Modelo I & $-1,2173$ & 7,9858 & 0,9717 & 0,9836 \\
Modelo II & $-6,6769$ & 17,5327 & 0,8127 & 0,9702 \\
Modelo III & 0,3766 & 19,5715 & 0,8792 & 0,9402 \\
\hline
\end{tabular}

Os Modelos I e II apresentaram valores negativos para MBE(\%), indicando subestimativa dos valores das respectivas irradiações $H_{G p}^{d}$ e $H_{d p}^{d}$, enquanto que o Modelo III apresentou valor positivo ou superestimativa do índice $K_{d p}^{d}$. Em termos percentuais absolutos de MBE, o Modelo que obteve melhor desempenho foi o III, mesmo com um valor intermediário entre os demais para o índice de correlação $\left(\mathrm{r}_{\mathrm{II}}=0,8792\right)$.

O Modelo I apresentou desempenho intermediário aos demais quanto a $\mathrm{MBE}$, enquanto que o pior desempenho foi obtido pelo Modelo II, sendo este comportamento esperado, pois o Modelo II foi ajustado através de valores de radiação difusa do espectro visível e total, que é dependente da concentração variável de moléculas suspensas na atmosfera e de partículas (aerossóis), as quais promovem o espalhamento ou difusão dos bandas do espectro da radiação, justificando assim o maior espalhamento dos pontos ao redor da reta ajustada na correlação que as demais correlações (que geraram os Modelos I e III), fato este comprovado através do mais baixo de todos valores obtidos para o índice de correlação $\left(\mathrm{r}_{\mathrm{III}}=0,8127\right)$. 

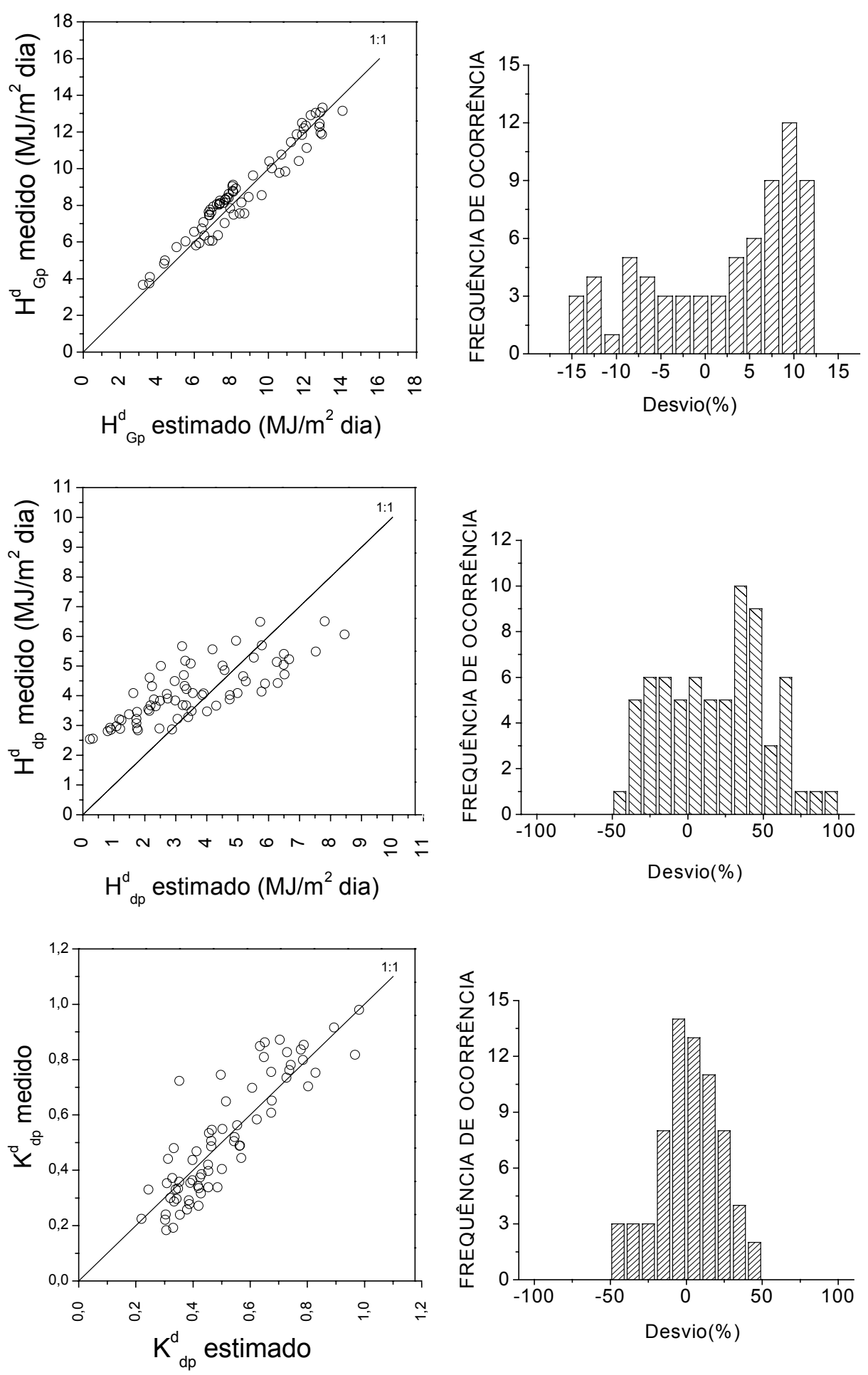

Figura 4. Validação dos Modelos I, II e III e respectiva frequência de ocorrência de desvios.

Os modelos II e III apresentam um desempenho inferior, com um pouco mais de 10 unidades percentuais a mais para RMSE (\%) e em torno de 0,10 unidades a menos para o índice $d$. Tal comportamento ocorre provavelmente devido ao fato das variáveis dependentes dos modelo II e III e independente do modelo II serem representadas pela fração 
difusa do espectro visível e total, como já mencionado anteriormente.

As correlações obtidas entre os valores estimados através dos modelos I, II e III e medidos das respectivas componentes $H_{G p}^{d}, H_{d p}^{d}$, e índice $K_{d p}^{d}$, bem como a reta ideal 1:1 (com $45^{0}$ de inclinação) estão expressos na Figura 4. A figura também apresenta a respectiva freqüência de ocorrência de desvios para cada um dos modelos.

A Figura 4 mostra que o menor espalhamento dos pontos ao redor da reta ideal (1:1) foi obtida na correlação entre os valores estimados e medidos de $H_{G p}^{d}$, enquanto que as demais correlações apresentaram um maior espalhamento, estando de acordo com os resultados obtidos pelo indicativo estatístico RMSE.

Os parâmetros estatísticos (coeficiente angular e índice de correlação) obtidos nas correlações entre Modelo I e $H_{G p}^{d}$, Modelo II e $H_{d p}^{d}$ e Modelo III e $K_{d p}^{d}$ estão expressos no Quadro 2.

Quadro 2. Coeficiente angular (b) e indicativos estatísticos de correlação entre Modelo I e $H_{G p}^{d}$, Modelo II e $H_{d p}^{d}$ e Modelo III e $K_{d p}^{d}$.

\begin{tabular}{cccc}
\hline Correlação & $\mathrm{b}$ & $\mathrm{r}$ & $\begin{array}{c}\mathrm{n}^{\circ} \text { de } \\
\text { observações }\end{array}$ \\
\hline $\begin{array}{c}\text { Modelo I } \\
\text { e } H_{G p}^{d}\end{array}$ & 1,0070 & 0,9684 & 70 \\
$\begin{array}{c}\text { Modelo II } \\
\text { e } H_{d p}^{d}\end{array}$ & 1,0065 & 0,8777 & 70 \\
$\begin{array}{c}\text { Modelo III } \\
\text { e } K_{d p}^{d}\end{array}$ & 0,9938 & 0,7817 & 70 \\
\hline
\end{tabular}

O Quadro 2 mostra valores próximos de 1 para os coeficientes angulares. Os valores acima de 1,00 obtidos para o coeficiente angular da reta ajustada entre Modelo I e $H_{G p}^{d}$ e Modelo II e $H_{d p}^{d}$ indicam que estes modelos estão subestimando os valores das respectivas irradiações $H_{G p}^{d}$ e $H_{d p}^{d}$, enquanto que o valor abaixo de 1,00 obtido para o coeficiente angular da reta ajustada entre o Modelo III e $K_{d p}^{d}$ indica a superestimativa dos valores de $K_{d p}^{d}$, fenômeno este já constatado pelo indicativo estatístico MBE. A correlação que apresentou o pior índice de correlação foi entre Modelo III e $K_{d p}^{d}$ $\left(r_{I I I}=0,7817\right)$, ao mesmo tempo que o Modelo III e $K_{d p}^{d}$ apresentaram uma frequência de ocorrência de desvios percentuais mais desejável, com distribuição de frequência tendendo a normal, e com o grande montante das observações apresentando desvio percentual zero.

\section{CONCLUSÕES:}

Pode-se estimar com considerável precisão as componentes $H_{G p}^{d}$ e $H_{d p}^{d}$ em função das respectivas componentes $H_{G}^{d}$ e $H_{d}^{d}$, através de modelos lineares, bem como a fração $K_{d p}^{d}$ em função de $K_{t}^{d}$ através de modelo polinomial de segundo grau.

Os modelos propostos tiveram um bom desempenho nos indicativos estatísticos. Quanto a MBE, os modelos I e III obtiveram valores percentuais absolutos menores que $1,22 \%$, enquanto que o Modelo II apresentou resultado ligeiramente pior, na ordem de 5,5 unidades percentuais a mais.

Os valores para RMSE obtidos pelos modelos I e II foram inferiores a 17,6\%, enquanto que $\mathrm{o}$ modelo III foram mais elevados, na ordem de 19,6\%, devido ao maior espalhamento ocorrido. $\mathrm{O}$ modelo I desempenhou-se melhor quanto a $\operatorname{RMSE}(\%)$, com 9,5 unidades percentuais a menos que o Modelo II e 11,6 unidades percentuais a menos que o Modelo III.

Os valores obtidos para $d$ foram elevados e próximos de 1 , e o uso conjunto deste indicativo com o RMSE(\%) permitiu uma análise mais precisa da performance dos modelos propostos, e através destes valores, o modelo I obteve o melhor desempenho, sendo seguido do modelo II e III. 


\section{REFERÊNCIAS BIBLIOGRÁFICAS:}

ALADOS, I.; ALADOS-ARBOLEDAS, L. Direct and diffuse photosynthetically active radiation: measurements and modelling. Agriculture and Forest Meteorology, New York, v. 93, n. 1, p. 27-38, 1999.

ALADOS. I.et al. Estimation of photosynthetically active radiation under cloudy conditions. Agriculture and Forest Meteorology, New York, v. 102, n. 1, p. 39-50, 2000.

ALVALÁ, R. C. S.; SILVA, F. W. Relação entre a radiação fotossinteticamente ativa e a irradiância de onda curta em área de policultivo na Amazônia. In: CONGRESSO BRASILEIRO DE METEOROLOGIA, 11., 2000, Rio de Janeiro. Anais... Rio de Janeiro: Congresso Brasileiro de Meteorologia, 2000. 1 CD-ROM. BRITTON, C. M.; DODD, J. D. Relationships of photosynthetically active radiation and shortwave irradiance. Agriculture

Meteorology, New York, v. 17, n. 1, p. 1-7, 1976.

BROCK, T. D. Calculating solar radiation for ecological studies. Ecological Modelling, Copenahgen, v. 14, n. 1, p. 1-19, 1981. FRISINA, V. A. et al. Equações de estimativas diárias das componentes global, difusa e refletida da radiação solar total e fotossinteticamente ativa (PAR) em estufa de polietileno. In: CONGRESSO BRASILEIRO DE METEOROLOGIA, 11., 2000, Rio de Janeiro. Anais...Rio de Janeiro: Congresso Brasileiro de Meteorologia, 2000. 1 CD-ROM. HASSIKA, P.; BERBIGIER, P. Annual cycle of photosynthetically active radiation in maritime pine forest. Agriculture and Forest Meteorology, New York, v. 90, n. 2, p. 157171, 1998.

HOWELL, T. A.; MEEK, D. W.; HATFIELD, J. L. Relationship of photosynthetically active radiation to shortwave radiation in the San Joaquin Valley. Agriculture Meteorology, New York, v. 28, n. 1, p. 157-175, 1983. IQBAL, M. An introduction to solar radiation. New York: Academic Press, 1983. $390 \mathrm{p}$.

KARALIS, J. D. Characteristics of direct photosynthetically active radiation.

Agriculture and Forest Meteorology, New

York, v. 48, n. 2, p. 225-234, 1989.

MCCREE, K. J. A solarimeter for measuring photosynthetically active radiation.

Agriculture Meteorology, New York, v. 3, n. 2, p. 353-366, 1966.

MONTEITH, J. L. Solar radiation and productivity in tropical ecossystems. Journal of Applied Ecology, London, v. 9, n. 4, p. 747766,1972.

PAPAIOANNOU, G.; PAPANIKOLAOU, N.; RETALIS, D. Relationships of photosynthetically active radiation and shortwave irradiance. Theoretical Applied Climatology, Vienna, v. 48, n. 1, p. 23-27, 1993.

SILVA, E. T.; ASMUS, M. L. A dynamic simulation model of the widgeon grass Ruppia maritma and its epiphytes in the estuary of the Patos Lagoon, RS, Brazil. Ecological

Modelling, Copenahgen, v. 137, n. 1, p. 161179, 2001.

STIGTER, C. J.; MUSABILHA, M. M. The conservative ratio of photosynthetically active to total radiation in the tropics. Journal of Applied Ecology, London, v. 19, n. 3, p. 853858, 1982.

WILLMOTT, C. J. On the validation of models. Physical Geography, London, v. 2, n. 1, p. 184-194,_1981.

YOCUM, C. S. et al. Photosynthesis under field conditions. VI. Solar radiation balance and photosynthetic efficiency. Agronomy Journal, Madison, v. 56, n. 2, p. 249-253, 1969. 